\title{
Genetic algorithms and solid state NMR pulse sequences
}

\author{
Matthias Bechmann* \\ Department of Chemistry \\ University of York \\ YO10 5DD York, UK \\ John Clark ${ }^{\dagger}$ \\ Department of Computer Science \\ University of York \\ YO10 5DD York, UK \\ Angelika Sebald ${ }^{\ddagger}$ \\ Department of Chemistry \\ University of York \\ YO10 5DD York, UK
}

17th December 2012

\begin{abstract}
The use of genetic algorithms for the optimisation of magic angle spinning NMR pulse sequences is discussed. The discussion uses as an example the optimisation of the $\mathrm{C} 7 \frac{1}{2}$ dipolar recoupling pulse sequence, aiming to achieve improved efficiency for spin systems characterised by large chemical shielding anisotropies and/or small dipolar coupling interactions. The optimised pulse sequence is found to be robust over a wide range of parameters, requires only minimal a priori knowledge of the spin system for experimental implementations with buildup rates being solely determined by the magnitude of the dipolar coupling interaction, but is found to be less broadbanded than the original $\mathrm{C} 72$ pulse sequence. The optimised pulse sequence breaks the synchronicity between r.f. pulses and sample spinning.
\end{abstract}

\section{Introduction}

Solid state magic angle spinning (MAS) NMR spectroscopy has become an indispensable and rather widely used tool for the characterisation of crystalline and non-crystalline powder materials. Amongst the magnetic interactions present, direct dipolar coupling plays a particularly prominent role owing to its direct relationship with internuclear distances $\left(\propto r^{-3}\right)$, making the measurement of direct dipolar coupling constants a highly attractive target from a structural point of view. In addition, NMR experiments employing cross correlations, multi-quantum excitation or polarisation transfer [62, 38, rely on the presence of direct dipolar coupling. Unsurprisingly, over the years much effort has been devoted to the development of MAS NMR pulse sequences that make direct dipolar coupling information accessible in an accurate, quantifiable and straightforward fashion [30, 10, 14, 12, 7, 2, 11].

\footnotetext{
*matthias.bechmann@york.ac.uk

†john@cs.york.ac.uk

‡angelika.sebald@york.ac.uk
} 
The theoretical description and development of MAS NMR experiments is typically accomplished by using the frameworks of average Hamiltonian (AHT) [67] or Floquet [40] theory. Application of these theories is difficult if the interaction of interest is not the dominating one or, as is the case of homonuclear direct dipolar coupling, renders the system Hamiltonian homogeneous. In such circumstances the description of the spin dynamic is only possible approximately. Consequently, pulse experiments derived by using such approximations are starting to fail if e.g. direct dipolar coupling is not the strongest interaction (long internuclear distances) and/or if other interactions, especially chemical shielding anisotropy (particularly at high external magnetic field strengths $\boldsymbol{B}_{0}$ ) are dominant. MAS NMR experiments that do not conform to the assumptions of lower-order AHT and Floquet theories can sometimes be improved by either using higher order approximations [26, 8, or by combinations of supercycling [42, 36, 37] and composite pulses [41. Furthermore, owing to the nature of these theoretical tools, MAS NMR pulse sequences typically consist of rotation-synchronised r.f. pulse trains. While the assumption of rotation synchronisation greatly facilitates the mathematical description, it excludes any non-synchronous experiments.

As an alternative approach numerical simulations of MAS NMR spin dynamics combined with various search algorithms have been used to improve existing experiments or to find new or improved pulse sequences. This approach has been made feasible by easy access to increasing computing power and advances in spectrometer hardware technology. Successful transfer of optimised numerical results to a real-world NMR spectrometer heavily relies on robust and finely tuneable hardware parameters. Such approaches have resulted in, for example, techniques such as strongly modulating pulses [17, 6, 48], a range of modified pulse sequences based on optimal control theory [19, 63, 49], or modulated decoupling schemes [59, 53. All these approaches have in common that they employ classical optimisation techniques based on Simplex- or Newton-type methods [15] which exploit the local shape of a particular fitness function in order to maximise performance. Numerical simulationbased optimisation approaches have also occasionally taken advantage of genetic algorithms (GA) instead of relying on classical optimisation [18, 22, 54, 24, 23.

Here we discuss that GAs offer a class of stochastic search algorithms that is able to explore parameter spaces more widely than classical algorithms and, hence, may be more suitable to find unconventional pulse sequences not easily accessible by other means. In particular, constraints can be chosen such that resulting pulse sequences are straightforward, enabling insight into the spin dynamics and, hence, offering a new starting point for the further development of theoretical descriptions. We describe the necessary requirements to use a GA in the search for improved MAS NMR experiments and apply this to a representative test case, the $\mathrm{C}_{2}^{1}$ pulse sequence [42] in the presence of large chemical shielding anisotropies (CSA) and small dipolar coupling interactions. The results are analysed with regard to robustness, possible trends and experimental verification. The properties and performance of the GA are compared to that of other (classical) search algorithms.

\section{Results and Discussions}

NMR experiments and pulse sequences in particular depend on multiple parameters and tend to have a modular structure. MAS NMR pulse sequences often display periodic repetition of basic r.f. pulse elements and the variation of these elements by modulation of a single parameter (commonly a r.f. pulse phase). This internal structure originates from the response of nuclear magnetic moments to external magnetic fields or sample rotation, and from the way theory is describing the spin response to these external perturbations [67, 40, often explicitly involving symmetry features [42]. Any numerical approach to pulse sequence design and/or optimisation needs to make the decision 
to either retain (some of) these symmetry principles or to ignore internal symmetry and structure in favour of an unbiased search approach. The first approach can reduce the dimension of the parameter space and results are likely to be closely linked to the underlying theoretical description of the spin dynamics but will require, to some extent, previous knowledge of the spin dynamics. The second approach is typically characterised by a potentially larger parameter space and a more complex structure with limited potential to provide insight into the spin dynamics. A hybrid approach between this two extremes is, for example, the unbiased optimisation of basic NMR pulses $\left(90^{\circ}, 180^{\circ}, \ldots\right)$ to compensate for experimental imperfections while keeping the overall pulse sequence unaltered: while making the pulse sequence experimentally more robust, this would not have the potential to improve the performance of the experiment in general.

Analysing the experimental performance of pulse sequences as a function of pulse sequence parameters by numerical simulations [5, 43, 39] commonly yields rugged error surfaces with multiple local minima, sharp and often singular resonance conditions, and areas of rather low variation inbetween. The exploration of such error landscapes in the search for new or improved pulse sequences is a difficult task for classical optimisation routines if the search starts far away from the global minimum, and if no conjectures about a likely location of optimum parameters can be made in advance. Stochastic search algorithms such as evolutionary and genetic algorithms (GA) [25, 61, 27] are much better placed to deal with this type of search scenario for a number of reasons. GAs provide high degrees of flexibility regarding the choice of fitness functions as compared with e.g. least-squares methods. GAs are independent of gradients with concomitant gains in speed especially if no analytically defined gradients exist. GAs can handle mixed parameters (such as continuous and discrete parameters) naturally well. All these features make the application of GAs an attractive alternative for purposes of NMR pulse sequence searches and optimisations.

In the following we demonstrate the application of a GA to the optimisation of a homonuclear dipolar recoupling MAS NMR pulse sequence, $\mathrm{C}_{2}^{1}$ [42. We use this pulse sequence as an example because it is widely used 42 and its practical advantages and disadvantages are thus well documented. Our objective is the optimisation of $\mathrm{C} 72 \frac{1}{2}$ performance for spin systems characterised by the simultaneous presence of large CSA and small dipolar coupling constants such that internuclear distances can be determined - a task not easily achieved by $\mathrm{C} 72 \frac{1}{2}$ in its original or some of its modified [13, 31] forms.

\subsection{GA optimisation of the $\mathrm{C} 7_{2}^{1}$ pulse sequence}

The $\mathrm{C} 7 \frac{1}{2}$ pulse sequence (see Figure 1) can be parametrised as

$$
\mathrm{C} N_{n}^{\nu}=\left\{\left[\left(\theta_{\mathrm{C}}\right)_{\phi_{\mathrm{C}}}\left(\theta_{\mathrm{C}}\right)_{\bar{\phi}_{\mathrm{C}}}\right]_{\phi_{\mathrm{C} N}}^{N}\right\}^{n_{\mathrm{C}}}
$$

with the space and spin winding number $n=2$ and $\nu=1$, the basic element $\mathrm{C}=\left(\theta_{\mathrm{C}}\right)_{\phi_{\mathrm{C}}}\left(\theta_{\mathrm{C}}\right)_{\bar{\phi}_{\mathrm{C}}}$, pulse flip angle $\theta_{\mathrm{C}}=2 \pi$, pulse phases $\phi_{\mathrm{C}}=0$ and $\bar{\phi}_{\mathrm{C}}=180, N=7$ iterations of the $\mathrm{C}$ elements, phase increment $\phi_{\mathrm{CN}}$ of the $\mathrm{C}$ elements, and $n_{c}$ iterations of $\mathrm{C} 7$ r.f. blocks. With pulse amplitude $\kappa_{\mathrm{C}}$ and duration $\tau_{\mathrm{C}}$, symmetry parameters $N, n$ and $\nu$ [42] demand the relations

$$
\begin{aligned}
\kappa_{\mathrm{C}} & =\frac{2 N}{n} \omega_{\text {rot }}=7 \cdot \omega_{\text {rot }} \\
\kappa_{\mathrm{C}} \tau_{\mathrm{C}} & =\theta_{\mathrm{C}} \\
\phi_{\mathrm{C} N} & =\frac{2 \pi \cdot \nu}{N}
\end{aligned}
$$




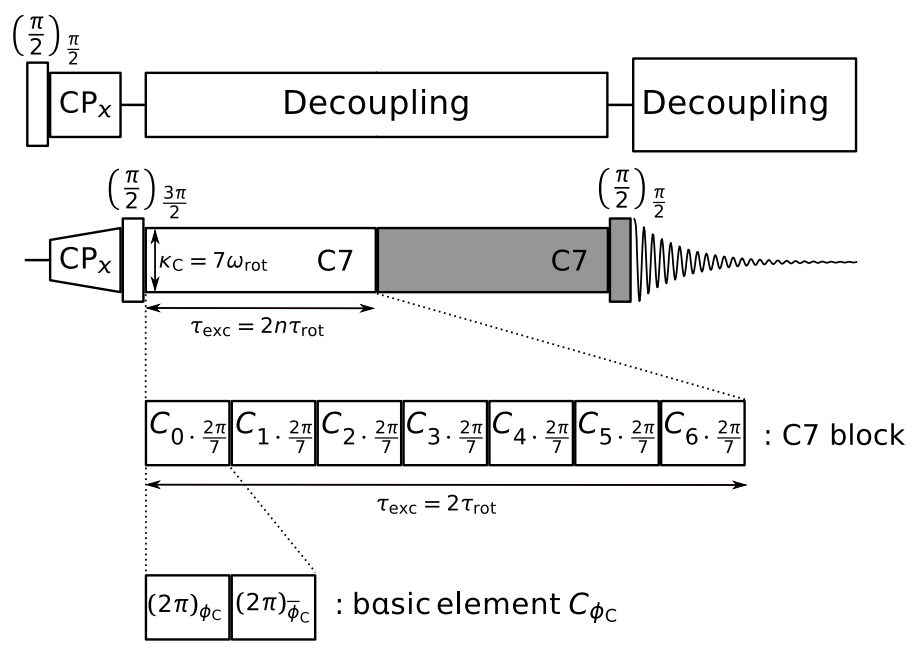

Figure 1: The $\mathrm{C} N_{n}^{\nu}=\mathrm{C} 7{ }_{2}^{1}$ pulse sequence for excitation and reconversion of double-quantum coherences [42]

For a given spinning speed $\omega_{\text {rot }}$ this parametrisation fixes all possible parameter values. For the optimisation we now keep the seven-step phase increment $\phi_{\mathrm{CN}}=\phi_{\mathrm{C} 7}$ and the number of pulses per C7 block $(14=7 \cdot 2)$. All other constraints are removed and $\kappa_{\mathrm{C}} \rightarrow \kappa_{1}, \kappa_{2}, \tau_{\mathrm{C}} \rightarrow \tau_{1}, \tau_{2}, \phi_{\mathrm{C}} \rightarrow \phi_{1}, \phi_{2}$ giving a pulse sequence parametrisation

$$
\mathrm{C} 7_{2 \mathrm{opt}}^{1}=\left\{\left[\left(\kappa_{1} \tau_{1}\right)_{\phi_{1}}\left(\kappa_{2} \tau_{2}\right)_{\phi_{2}}\right]_{\phi_{\mathrm{C} 7}}^{7}\right\}^{n_{\mathrm{C}}}
$$

with seven variable parameters $\left(\kappa_{1}, \kappa_{2}, \tau_{1}, \tau_{2}, \phi_{1}, \phi_{2}, n_{\mathrm{C}}\right)$.

Double-quantum filtration (DQF) experiments are constructed using Equation 5 in the usual way (see Figure 1)

$$
F I D=\mathrm{C}_{2}^{1}{ }_{\text {opt }}-\mathrm{DQF}-\left(\mathrm{C} 7_{2 \text { opt }}^{1}\right)_{90}-\text { acq }
$$

with a $90^{\circ}$ r.f. phase shift during reconversion to achieve absorptive lineshapes. This pulse-sequence template with seven variable parameters is now interfaced with the GA to generate candidate solutions for improved NMR experiments.

The GA simulates the selection from a population of candidate solutions with the goal of evolving the population to a more fit and more diverse next generation. This evolution process is accomplished by applying genetic operators like inheritance, mutation, selection and crossover to an existing population of candidate solutions. In order to use a GA it is necessary to represent the solution to a given problem (phenotype) as a genome or chromosome (genotype) 20. Overall every genetic algorithms requires the implementation of three crucial components: 1) an objective/fitness/cost function, 2) the definition and implementation of the genetic representation/encoding of the problem description and 3) the definition and implementation of the genetic operators:

1. The objective function $f$ is calculated from Equation 6 as

$$
f=1-F I D(1)
$$


This takes the normalised, first-point intensity in the FID as representative of the whole integrated spectral area and hence the DQF efficiency of the pulse sequence. The normalised spectral intensity $F I D(1)$ can assume positive and negative values $[-1,1]$ in homonuclear recoupling DQF experiments [9, but for the operation of the algorithm it is advantageous to only have positive fitness values. This is achieved by the form of Equation 7 where more fit candidates have smaller values $f$. Experimentally, this choice of fitness function means that here we concentrate on improved efficiency of the pulse sequence. In some instances the single-quantum elements in the final density matrix can be an alternative measure of DQF efficiency.

2. The parameters in Equation 5 control the performance of the NMR pulse experiment. Therefore, these parameters are the real-world parameters of our search problem and are referred to as phenotype of the problem description. GA operators do not work on this description, but on its genetic encoding, the genotype. This genetic representation of the pulse sequence is achieved by mapping each pulse-sequence parameter to a bit-string representation, the genes. Together these genes form the genome/chromosome of the search problem. The bit-string mapping is achieved by defining decimal boundary values and a bit depth which controls the range and resolution over which the parameter can vary during the optimisation process (Figure 2). A suitable choice of bit depth in relation to the boundary values allows to control encoding of integer and float pulse-sequence parameters.

3. Different pulse sequences correspond to different genomes and together form a population of a given size. Every member of this population is a candidate solution to the search problem. A population is evolved to the next generation by three genetic operations. Individuals/parents are selected for later crossover/mating using roulette-wheel selection, also called fitness-proportionate selection. Individuals are more likely to be selected the fitter they are according to their objective function. Elitism [66] is applied to avoid that the stochastic selection process misses the best candidate of a population. The crossover probability $p_{\mathrm{c}}$ controls how many individuals of a population are selected to mate. Here one-point crossover is used as shown in Figure 3, Mutation is the process of changing elements in the genome by a random process. A random flip mutator is used that toggles the bit value in a gene according to a mutation probability $p_{\mathrm{m}}$ (Figure 4).

Various GAs can be created by the way the components 1-3 are implemented and combined. The overall operation of the GA follows the steps shown in Figure 5 .

- An initial population of $N_{\mathrm{P}}$ pulse sequences is generated with random pulse sequence parameter values from within the allowed parameter ranges. The random assignment of parameter values in the initial population ensures a diverse distribution of pulse sequences over the complete search space.

- The fitness function value $f$ of every pulse sequence is calculated by simulating the respective NMR experiment. The fitness values represent point intensities in the overall search space. Individual pulse sequences are selected for mating based on their fitness value, and deemed unfit pulse sequences are discarded. This generates a subpopulation of very fit individuals but also reduces diversity amongst the pulse sequences.

- Pairs of this subpopulation mate via the crossover genetic operation until a new population of pulse sequences is generated. 


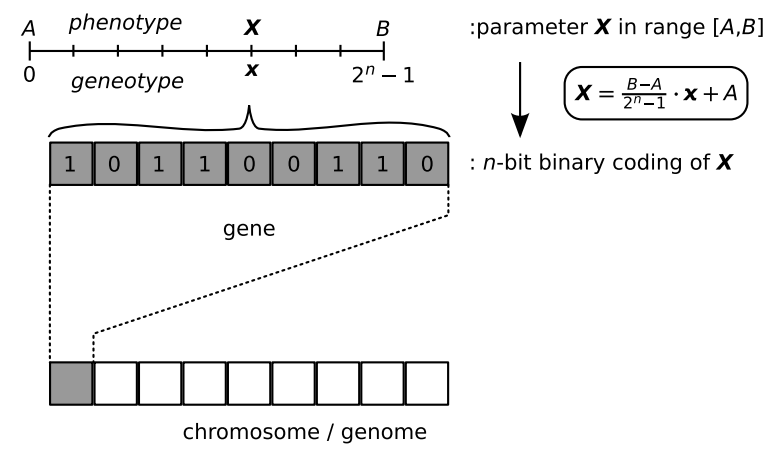

Figure 2: Pulse sequence parameter $\boldsymbol{X}$ is variable over interval $[A, B]$. It is binary encoded as a bit-string $\boldsymbol{x}$ of size $n$ to form a gene. Together with the other encoded parameters the genes form the genome or chromosome of our problem.

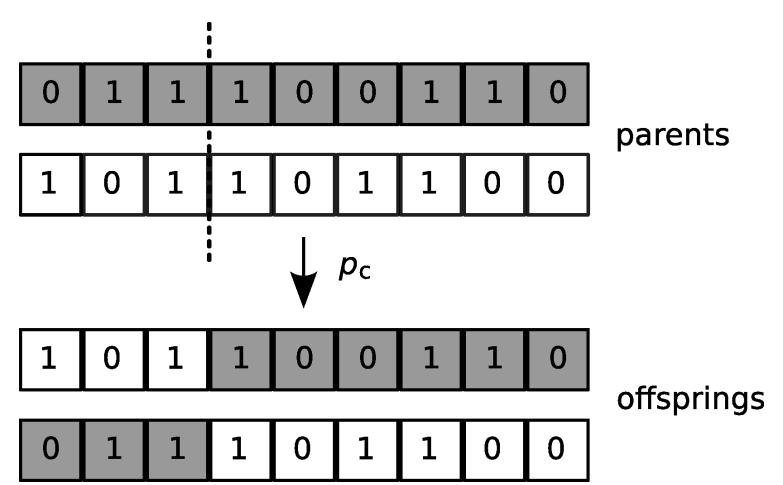

Figure 3: One-point crossover at a random position.

\begin{tabular}{|c|c|c|c|c|c|c|c|c|}
\hline 0 & 1 & 1 & 1 & 0 & 0 & 1 & 1 & 0 \\
\hline \multicolumn{9}{|c|}{$p_{\mathrm{m}} \downarrow$ mutation } \\
\hline 0 & 1 & 1 & 0 & 0 & 0 & 1 & 1 & 0 \\
\hline
\end{tabular}

Figure 4: Flip mutation genetic operator. 


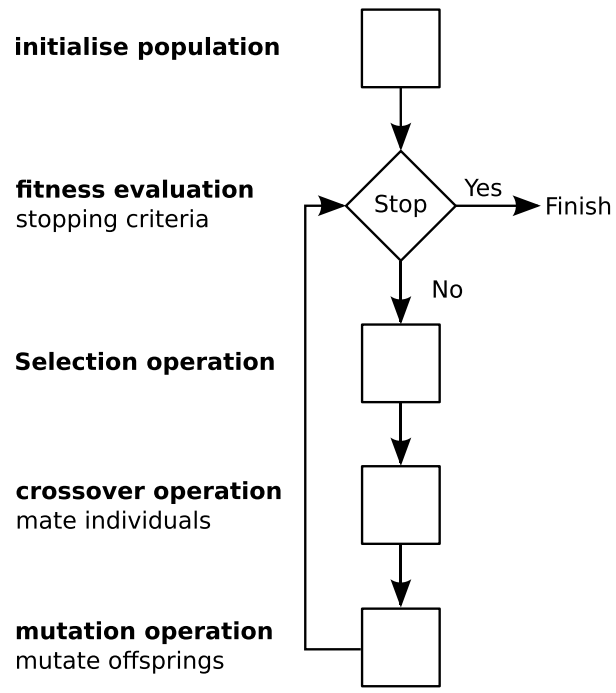

Figure 5: Flowchart of the procedure of a genetic algorithm.

- Members of this new population undergo the mutation operation and afterwards represent the final state of a new generation of pulse sequences. Mutation is the mechanism that can increase diversity by being able to change one genome to any other possible genome. Dependent on how frequently mutation occurs, this drives exploration of the search space.

- Fitness evaluation is used to decide on how to progress in the algorithm. Either the current population is evolved to a new generation, or the algorithm stops. Stopping criteria can be defined very flexibly and are typically based on optimum fitness and/or a predefined number of generations.

The performance of a GA not only depends on the implementation of the genetic operators, but is also susceptible to the tuning of the parameters: population size $N_{\mathrm{p}}$, crossover probability $p_{\mathrm{c}}$, mutation probability $p_{\mathrm{m}}$, and the definition of the fitness function [21. Their optimum settings are dependent on the goal of the search task at hand. In the context of finding new and improved NMR pulse sequences one would usually like a GA behaviour that explores the parameter search space widely during the early generations of the algorithm run. This gives a good chance of collecting a set of diverse and fit pulse sequences. Later in the run, more localised optimisation towards improving the best of these fit candidates is preferred.

\subsection{Implementation and exploration of GA optimisation of $\mathrm{C} 7 \frac{1}{2}$}

The performance of GA-generated $\mathrm{C} 7{ }_{2}^{1}$-derived pulse sequences is tested by using the spin system parameters of $1,4{ }^{13} \mathrm{C}_{2}$-mono-ammonium maleate, $\mathbf{1}$ (see Figure 6) as input to the numerical spindynamics simulations [3]. The ${ }^{13} \mathrm{C}$ spin pair in $\mathbf{1}$ displays a large CSA, no isotropic chemical shielding difference, and a relatively small dipolar coupling constant $b_{14} / 2 \pi=-216 \mathrm{~Hz}$. These are characteristics that typically hinder the $\mathrm{C}_{2}^{1}$ pulse sequence from achieving an optimum DQF efficiency of $\approx 73$ percent and lead to DQF-buildup rates that do not solely dependent on the 

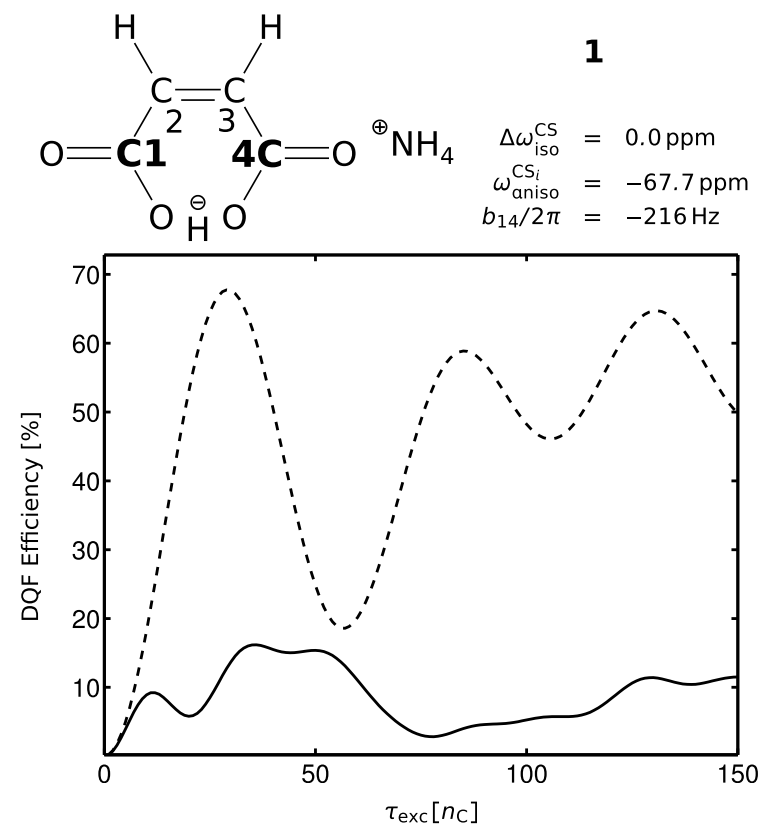

Figure 6: Simulated $\mathrm{C} 72 \mathrm{DQF}$ buildup curves based on the known spin-system parameters [16] of $1,4-{ }^{13} \mathrm{C}_{2}$-mono-ammonium maleate, $\mathbf{1}$. The lower curve $(-)$ includes CSA, the other curve (---) assumes absence of CSA

dipolar coupling constant $b_{14}$ (see Figure 6): for 1 , a maximum of only $\approx 16$ percent DQF efficiency is reached for an excitation period of $7.1 \mathrm{~ms}$, which is considerably longer than the optimum duration of $5.7 \mathrm{~ms}$ predicted from the value of $b_{14} / 2 \pi=-216 \mathrm{~Hz}$.

Due to the strong effect of CSA on the DQF buildup curve (Figure 6) it is a good strategy to initially investigate the effect of every optimisation parameter individually (durations, amplitudes, phases). A single-parameter sensitivity test is either accomplished by step-wise scanning the parameter over a defined range or by applying the GA with only one variable parameter. All other parameters are fixed to the default values of $\mathrm{C}_{2}^{1}$. This approach also allows to determine appropriate parameter-interval limits and bitstring sizes (Figure 2) for consecutive full optimisation runs.

The most sensitive parameter under single parameter optimisation are the pulse durations $\tau_{1}$ and $\tau_{2}$, causing an increase in DQF efficiency from $\approx 9$ percent of standard $\mathrm{C} 7{ }_{2}^{1}$ to $\approx 59$ percent. This is followed by the pulse amplitudes $\kappa_{1}$ and $\kappa_{2}$, optimisation of which increases DQF efficiency to $\approx 23$ percent. Phases $\phi_{1}$ and $\phi_{2}$ do not cause any substantial increase in DQF efficiency (compare Table 1). A step-wise increase of the number of simultaneously optimised parameters, ordered by decreasing sensitivity in single parameter scans, provides optimum parameters as shown in Table 1. As expected, simultaneous optimisation of all parameters results in the overall best DQF efficiency (65.5 percent). Comparing this to the DQF efficiency, achieved by solely optimising $\tau_{1}$ and $\tau_{2}$, suggests that enhancements are predominantly due to changes in pulse durations.

The experimental verification of DQF-efficiency enhancement by adjusting the pulse duration $\tau_{1}=\tau_{\mathrm{C}}+\Delta \tau_{1}$ is shown in Figure 7 An enhanced DQF-efficiency buildup of the optimised pulse 


\begin{tabular}{cccccccccc}
\hline run & $\tau_{1} /[\mathrm{ps}]$ & $\tau_{2} /[\mathrm{ps}]$ & $\kappa_{1} /[\mathrm{Hz}]$ & $\kappa_{2} /[\mathrm{Hz}]$ & $\phi_{1} /\left[^{\circ}\right]$ & $\phi_{2} /\left[^{\circ}\right]$ & $n_{\mathrm{CN} N} /[\#]$ & $f /[0-2]$ & $\mathrm{DQF} /[\%]$ \\
\hline \hline $\mathrm{C} 72$ & 14.00 & 14.00 & 71428 & 71428 & 0.00 & 0.00 & 31 & 0.91 & 8.69 \\
\hline GA & 14.36 & & & & & & & 0.44 & 55.69 \\
GA & 13.97 & 14.27 & & & & & & 0.41 & 59.28 \\
GA & & & 45232 & 76074 & & & & 0.69 & 31.14 \\
GA & & & & & -82.83 & 144.28 & & 0.87 & 13.46 \\
GA & 13.02 & 15.34 & 76643 & 66700 & & & & 0.42 & 57.93 \\
GA & 14.05 & 14.39 & 78477 & $=\kappa_{1}$ & & & 31 & 0.42 & 58.06 \\
GA & 12.83 & 16.02 & 77838 & 64648 & -9.94 & -6.29 & & 0.39 & 60.80 \\
GA & 14.63 & 15.04 & 76461 & 76221 & -1.86 & -8.18 & 34 & 0.35 & 65.45 \\
\hline
\end{tabular}

Table 1: Optimum parameter sets from GA optimisation. Empty cells are set to the default values of $\mathrm{C}_{2}^{1}$; Fitness $f$ and DQF efficiencies are best of 30 consecutive GA runs each limited to 1500 fitness evaluations distributed over population sizes of 50 individuals evolved for 30 generations. Crossover probability was $p_{\mathrm{c}}=0.6$ and mutation probability $p_{\mathrm{m}}=0.01$. The GA utilised parameter boundaries centred at $\mathrm{C} 72$ default values $\left(\Delta \tau_{i}= \pm 5 \mu \mathrm{s}, \Delta \kappa_{i}= \pm 7142 \mathrm{~Hz}, \Delta \phi_{i}= \pm 10^{\circ}, \Delta n_{\mathrm{C} 7}=\right.$ $\pm 20)$ and a bitstring length of 16 for floats.

a)
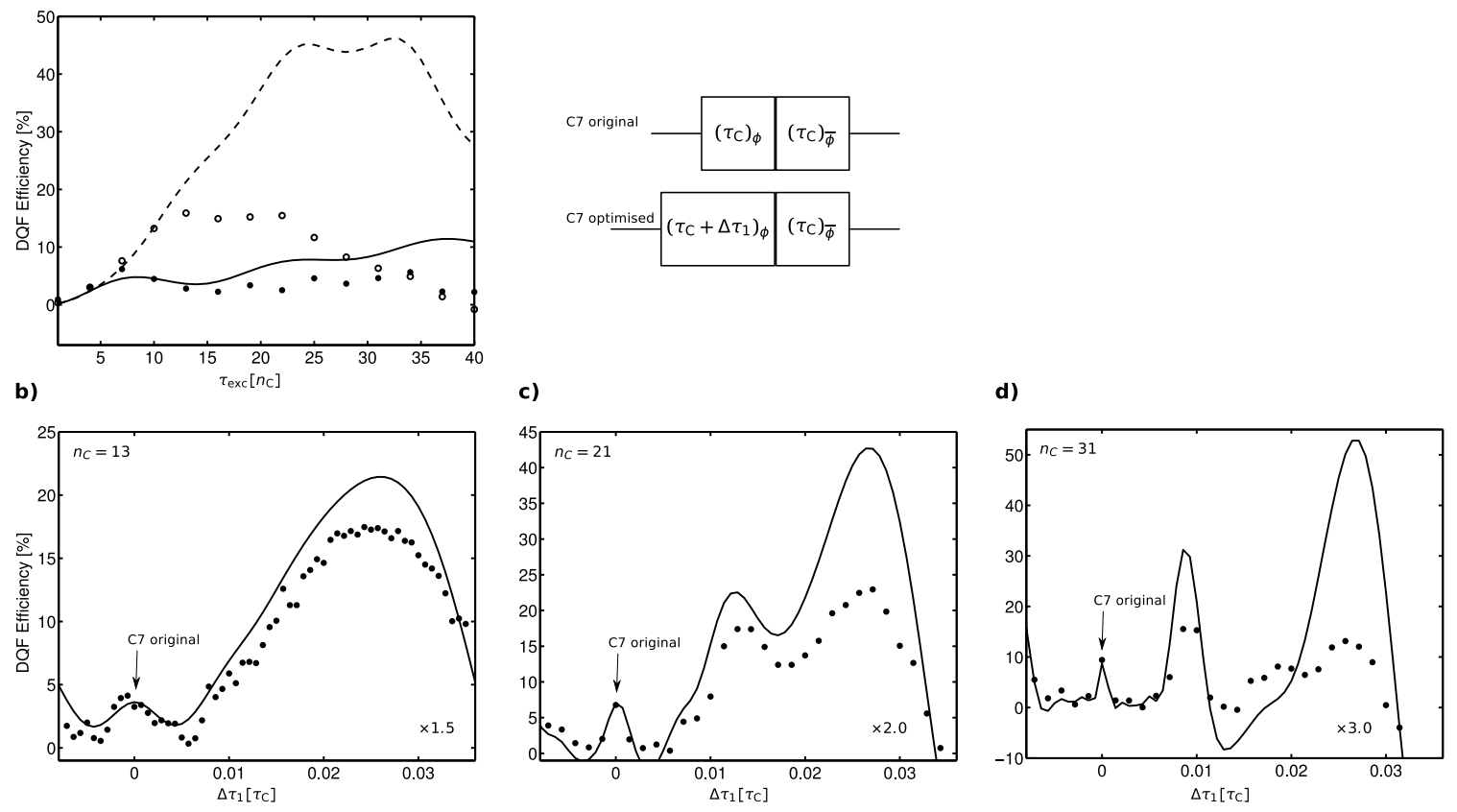

Figure 7: DQF efficiencies of optimised $\mathrm{C} 72$ pulse sequences. a) simulated DQF buildup curves as a function of $n_{\mathrm{C}}$ of original $\Delta \tau_{1}=0(-)$ and optimised $\Delta \tau_{1}=0.026 \tau_{\mathrm{C}}(--) \mathrm{C} 7_{2}^{1}$ together with basic $\mathrm{C}$ elements depicting the modifications. Also plotted are the respective experimental efficiencies $(\bullet \bullet)$ and $(\circ \circ \circ)$. b) -d) DQF-efficiency as a function of pulse duration change $\Delta \tau_{1}$ for different numbers $n_{\mathrm{C}}$ of $\mathrm{C} 7$ blocks 
sequence as compared to standard $\mathrm{C}_{2}^{1}$ can be verified experimentally (Figure $7 \mathrm{~h}$ ). However, the agreement between simulation and experiment is only good for excitation times up to ca. $n_{\mathrm{C}}=21$. The numerically predicted overall maximum DQF efficiency at $n_{\mathrm{C}}=31$ is not reached. This degradation of experimental performance with increasing pulse sequence length is most likely due to experimental imperfections such as accumulated (very minor) pulse maladjustments over the large number of pulses (868 at $n_{\mathrm{C}}=31$ ) and/or thermal heating of the NMR probe caused by the continuous r.f. irradiation over an extended period of time (e.g. $12.5 \mathrm{~ms}$ for $n_{\mathrm{C}}=31$ ) in the ${ }^{13} \mathrm{C}$ channel. The change in DQF efficiency as a function of pulse variation $\Delta \tau_{1}$ displays two clear efficiency maxima for both the original $\mathrm{C} 7_{2}^{1}$ and the optimised sequence at $\Delta \tau_{1}=0$ and $\Delta \tau_{1}=$ $0.026 \tau_{\mathrm{C}}$ respectively (Figure $7 \mathrm{~b}-\mathrm{d}$ ). These two maxima occur at constant positions for different excitation times, and the overall maximum DQF efficiency is always reached at $\Delta \tau_{1}=0.026 \tau_{\mathrm{C}}$. Both experiments and simulations generate this overall behaviour. For shorter excitation times $\left(n_{\mathrm{C}}<21\right)$ there is very good agreement between experiments and simulations. The optimised pulse sequence is able to experimentally achieve higher DQF efficiencies even for non-optimum durations of the excitation/reconversion periods (e.g. for $n_{\mathrm{C}}>21$ ) the efficiency is six times that of $\mathrm{C} 72_{2}^{1}$.

Next we will inspect the effect of variations of pulse durations $\tau_{1}$ and $\tau_{2}$ on DQF efficiency in somewhat more detail. Given that these are by far the two most dominant optimisation parameters, numerical analysis and visualisation of their effects is much facilitated as we can safely exclude the remaining, much less sensitive parameters from this inspection.

The symmetry rules at the core of $\mathrm{C} 7_{2}^{1}$ require the duration of a $\mathrm{C} 7$ block $\tau_{\mathrm{C} 7}$ to be an integer multiple of the rotor period $\tau_{\mathrm{C} 7}=7 \cdot\left(2 \tau_{\mathrm{C}}\right)=2 \tau_{\text {rot }}$. One $\mathrm{C} 7$ block duration $\tau_{\mathrm{C} 7}$ is filled with fourteen stacked $2 \pi$ pulses of duration $\tau_{\mathrm{C}}$. A change in the pulse duration by $\Delta \tau_{1}$ and $/$ or $\Delta \tau_{2}$ with $\Delta \tau_{1}+\Delta \tau_{2} \neq \bmod 2 \tau_{\mathrm{C}}$ renders the $\mathrm{C} 7$ block duration $\tau_{\mathrm{C} 7}^{\prime}=\tau_{\mathrm{C} 7}+\Delta \tau_{\mathrm{C} 7}$ asynchronous with the rotor period by

$$
\Delta \tau_{\text {rot }}=\frac{\Delta \tau_{\mathrm{C} 7}}{2}=\frac{7}{2}\left(\Delta \tau_{1}+\Delta \tau_{2}\right)
$$

The very small optimum values of $\Delta \tau_{1}$ (Figure 7) lead to quite small deviations from synchronicity with the excitation time $\tau_{\mathrm{exc}}=\tau_{\mathrm{C} 7} n_{\mathrm{C}}$ changing to

$$
\tau_{\mathrm{exc}}+\Delta \tau_{\mathrm{exc}}=\tau_{\mathrm{C} 7} n_{\mathrm{C}}+\Delta \tau_{\mathrm{C} 7} n_{\mathrm{C}}
$$

Further, changing the pulse duration also alters the pulse flip angle from $\theta_{\mathrm{C}}=2 \pi$ to

$$
\theta=\theta_{\mathrm{C}}+\Delta \theta
$$

Changes of the basic C-element pulse duration $\tau_{\mathrm{C}}$ by varying $\Delta \tau_{1}$ and $\Delta \tau_{2}$ affects the DQF efficiency as can be seen in the contour plots in Figure 8 In the absence of CSA (Figure 8k) a parabolically shaped area of high DQF efficiency can be identified, where $\Delta \tau_{1}=\Delta \tau_{2}=0$ corresponds to the vertex of the parabola and also the overall maximum DQF efficiency. This is in perfect agreement with the predicted optimal behaviour of $\mathrm{C}_{2}^{1}$. In the presence of CSA (Figure 8b), the high DQF-efficiency parabola and a line

$$
\Delta \tau_{1}+\Delta \tau_{2}=0
$$

of very low DQF efficiency occur, intersecting at the original $\mathrm{C} 72$ condition. Additional local efficiency maxima occur for the conditions $\Delta \tau_{1}+\Delta \tau_{2}=\frac{\tau_{\mathrm{C}}}{2} k+\frac{\tau_{\mathrm{C}}}{2} l$ with $k+l=4 j ; j, k, l \in \mathbb{N}$ but these are very strongly affected by CSA (see supplementary material)

Figure 9 displays the effect of pulse variations $\Delta \tau_{1}$ and excitation times $\tau_{\text {exc }}$ on DQF efficiency. 
a)

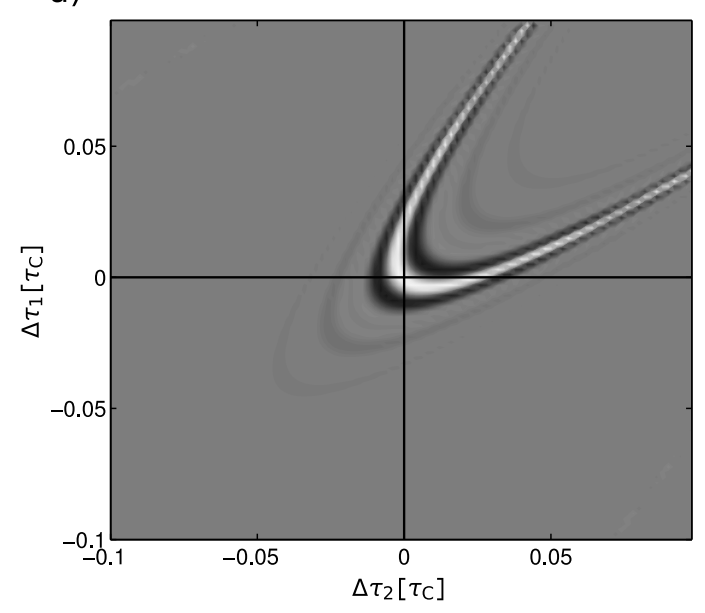

b)

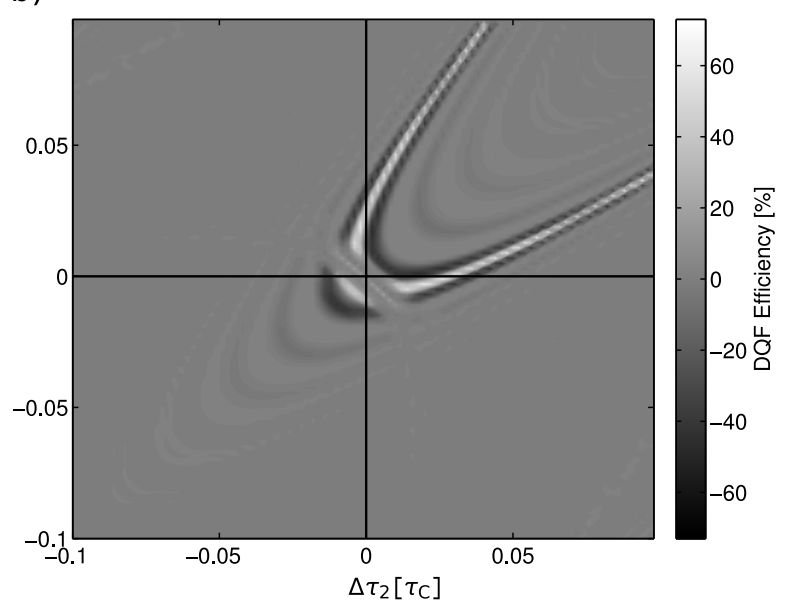

Figure 8: Two-dimensional contour plots of DQF efficiency as function of $\Delta \tau_{1}$ and $\Delta \tau_{2}$; crosshair marking indicates values of $\mathrm{C}_{2}^{1}$. Contour plots are based on the spin-system parameters of $\mathbf{1}$, using the excitation time of maximum DQF efficiency $\left(\tau_{\mathrm{exc}}=31 \tau_{\mathrm{C} 7}\right)$. a) Assuming absence of CSA, b) assuming presence of CSA.

a)

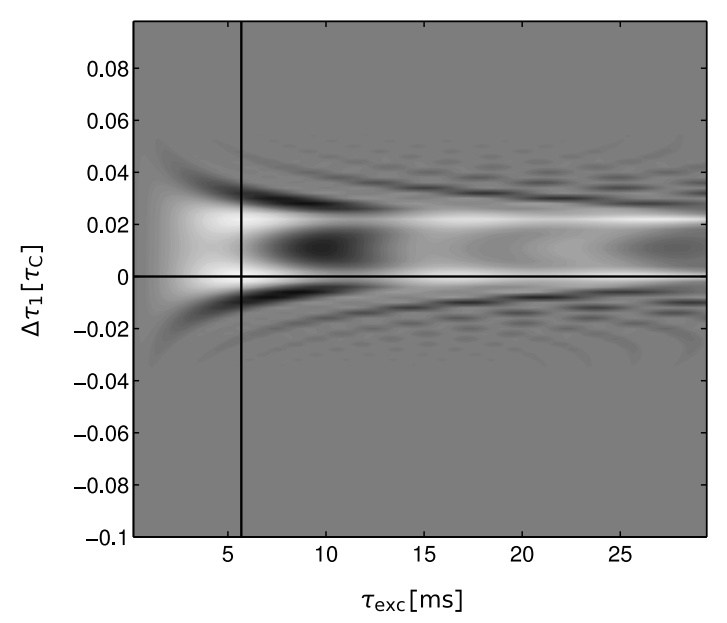

b)

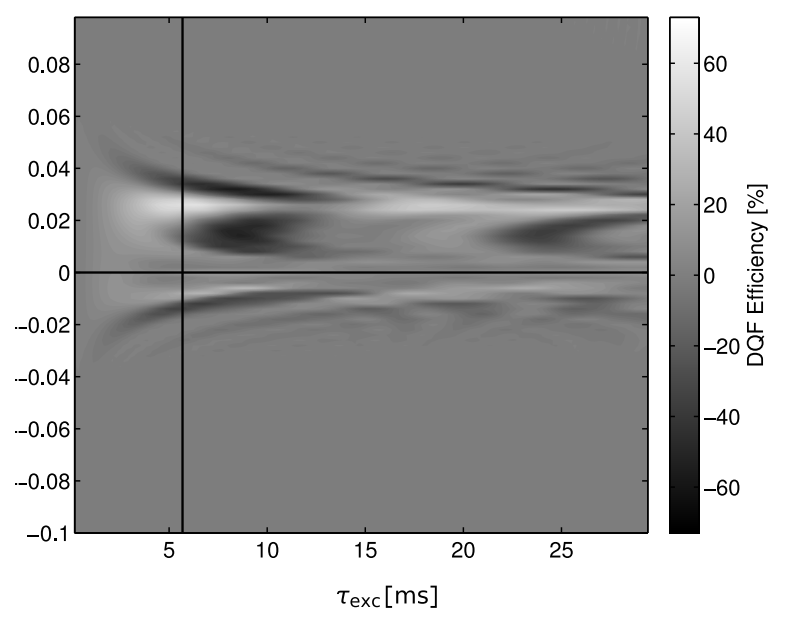

Figure 9: Two-dimensional contour plots of DQF efficiency as function of $\Delta \tau_{1}$ and $\tau_{\text {exc }}$; crosshair marking indicates values of $\mathrm{C}_{2}^{1}$. Contour plots are based on the spin-system parameters of 1 . a) Assuming absence of CSA, b) assuming presence of CSA. 

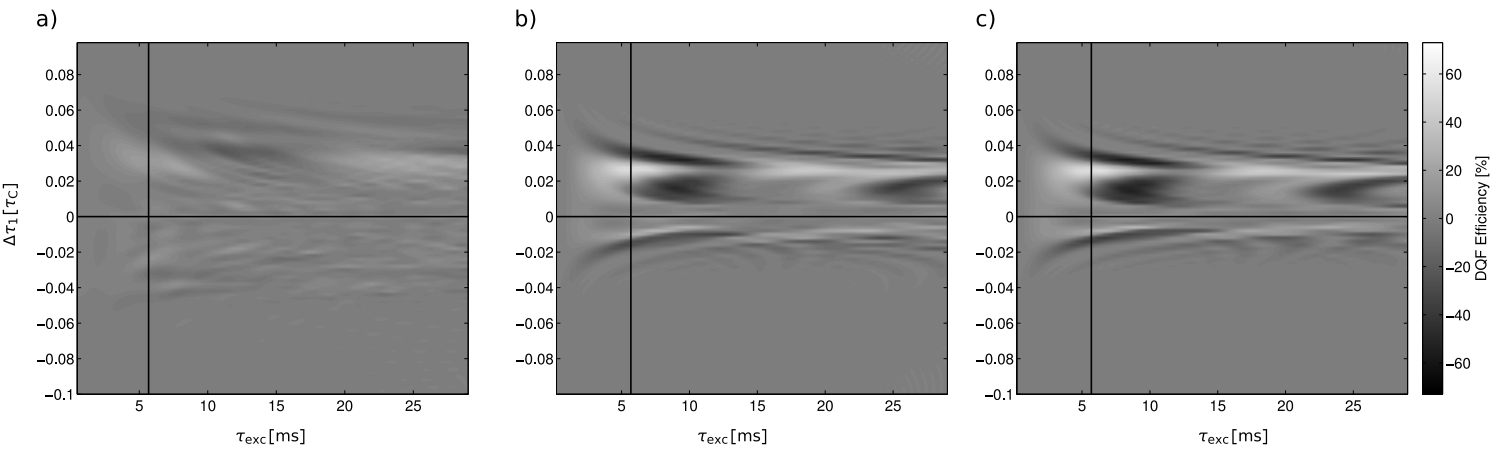

Figure 10: Spinning frequency dependence of DQF buildup of 1 as a function of excitation time $\tau_{\text {exc }}$ and pulse duration $\Delta \tau_{1}:$ a) $\omega_{\text {rot }}=0.39 \cdot \omega_{\text {aniso }}^{\mathrm{CSA}}$, b) $\omega_{\text {rot }}=0.88 \cdot \omega_{\text {aniso }}^{\mathrm{CSA}}$, c) $\omega_{\text {rot }}=0.99 \cdot \omega_{\text {aniso }}^{\mathrm{CSA}}$;

Horizontal slices through these contour plots represent the usual DQF-efficiency buildup curves as are e.g. shown in Figure 6 for $\Delta \tau_{1}=0$. For vanishing CSA (Figure 9 ) two symmetrically shaped horizontal bands of efficient DQF buildup reach DQF values close to the theoretical optimum at identical buildup rates and, therefore, reach maximum at identical excitation times $\tau_{\text {exc }}^{\max }$. The bands are centred at $\Delta \tau_{1}=0$ and at

$$
\Delta \tau_{1} \approx 0.022 \tau_{\mathrm{C}}
$$

This situation changes if substantial CSA is present (Figure 9b). There are still two bands present but the symmetry is broken. The band at $\Delta \tau_{1}=0$ displays large degradation of DQF efficiency (see Figure 6) and the (relative) maximum DQF efficiency is reached for a different excitation time. The second band is largely unaffected by the presence of CSA, featuring the same behaviour as the two bands in the scenario without CSA. Hence, for this band the DQF buildup rate is determined only by the dipolar coupling interaction, even in the presence of substantial CSA (Vertical slices of the contour plot, depicting this behaviour as a function of $\Delta \tau_{1}$, are shown in Figure $7 \mathrm{~b}-\mathrm{d}$ ).

In order to exploit these CSA-independent DQF-efficiency buildup conditions it is important to be able to predict their location precisely. In this respect the stable condition of Equation 12 is very useful. Figure 10 further illustrates this stability for the DQF-efficiency buildup as a function of $\Delta \tau_{1}$ and $\tau_{\text {exc }}$ for three different spinning speeds $\omega_{\text {rot }}$ in the presence of CSA. It is apparent that for spinning speeds less than ca. $0.9 \cdot \omega_{\text {aniso }}^{\mathrm{CSA}}$ the overall DQF efficiency is fairly low. For spinning speeds equal to or larger than the CSA interaction the desirable stable behaviour according to Equation 12 (Table 2) is observed. The data in Table2 further confirm this robust, CSA-independent behaviour.

Another important characteristic of recoupling MAS NMR experiments, besides interactionselective buildup rates, is their behaviour in terms of broadbandedness [4]. In the absence of CSA the $\mathrm{C} 72$ pulse sequence is able to generate high DQF efficiency over a full-width-at-halfmaximum range of $9.5 \mathrm{kHz}$, symmetrically placed around the transmitter frequency (see Figure 11). The presence of CSA not only reduces considerably the height of this profile, it also breaks the symmetry of the profile around the transmitter frequency and shifts the maximum height / maximum efficiency away from the transmitter frequency. In practical applications, this can make it rather difficult to choose appropriate experimental conditions. Our GA optimised pulse sequence restores the height and symmetry of the excitation profile around the transmitter frequency but is much more narrowbanded than the original $\mathrm{C}_{2}^{1}$ pulse sequence. This makes our optimised pulse 


\begin{tabular}{ccccc}
\hline \multirow{2}{*}{$\omega_{\text {rot }} /[\mathrm{Hz}]$} & \multicolumn{2}{c}{ without CSA } & \multicolumn{2}{c}{ with CSA } \\
\cline { 2 - 5 } & $\tau_{\mathrm{exc}}^{\max } /[\mathrm{ms}]$ & $\Delta \tau_{1}^{\max } /\left[\tau_{\mathrm{C}}\right]$ & $\tau_{\mathrm{exc}}^{\max } /[\mathrm{ms}]$ & $\Delta \tau_{1}^{\max } /\left[\tau_{\mathrm{C}}\right]$ \\
\hline 4000 & 5.500 & 1.022 & $*)$ & $*)$ \\
9000 & 5.777 & 1.022 & 5.403 & 1.026 \\
10204 & 5.684 & 1.022 & 6.354 & 1.026 \\
\hline
\end{tabular}

Table 2: Spinning frequency independence of the DQF-efficiency buildup maximum for the optimised $\Delta \tau_{1}$. Coordinates $\tau_{\mathrm{exc}}^{\max }$ and $\Delta \tau_{1}^{\max }$ are given: assuming absence of CSA and presence of CSA (compare Figure 9 and 10). ${ }^{*}$ ) indicates absence of a clear maximum at low spinning speeds.

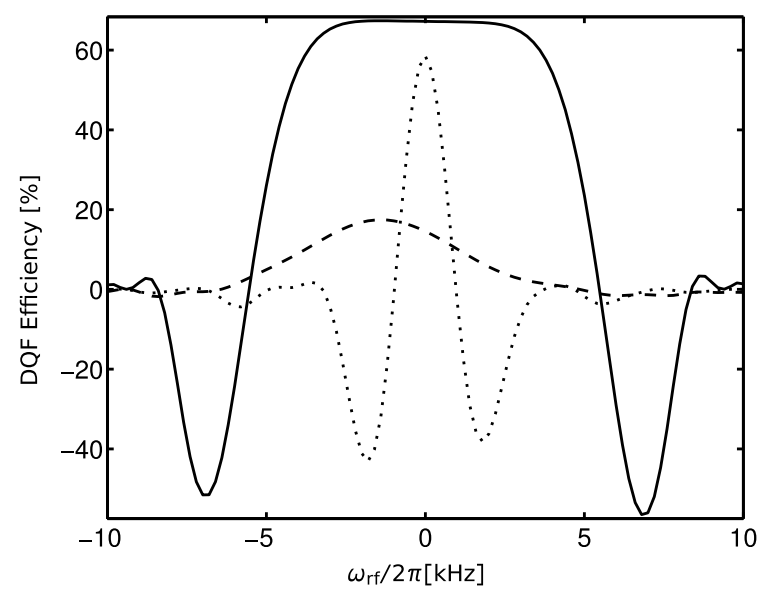

Figure 11: Transmitter-offset dependence of DQF efficiency for 1 at maximum DQF efficiency $\left(\tau_{\text {exc }}=31 \tau_{\mathrm{C} 7}\right)$ for: ideal $\mathrm{C}_{2}^{1}$ (absence of $\left.\mathrm{CSA}-\right)$, original $\mathrm{C}_{2}^{1}$ (including CSA ---), optimised pulse sequence parameters (including CSA $\cdots$ ). 


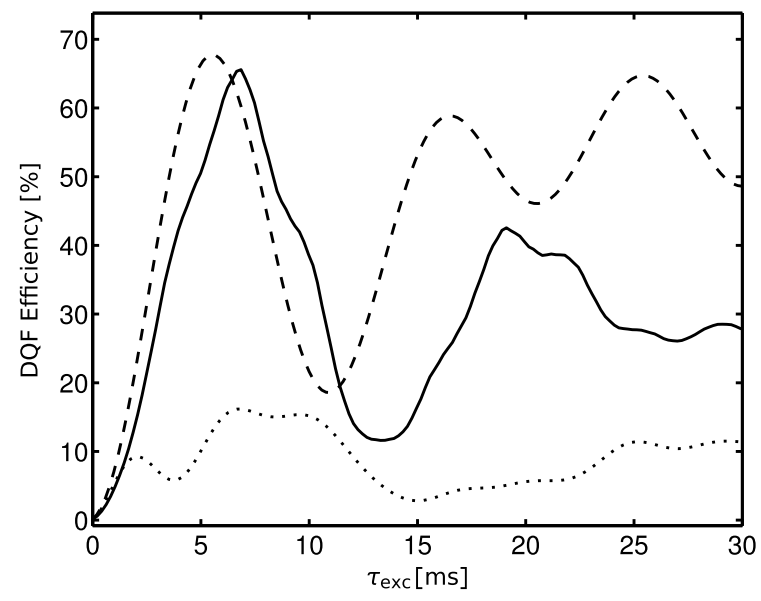

Figure 12: Simulated DQF efficiency buildup for 1 for: an ideal $\mathrm{C}_{2}^{1}$ buildup (absence of CSA ---), original $\mathrm{C}_{2}^{1}$ buildup (including CSA $\cdots$ ), all pulse sequence parameters $\left(\kappa_{2}, \tau_{1}, \tau_{2}, \phi_{1}, \phi_{2}, n_{\mathrm{C}}\right)$ optimised (including CSA -).

sequence a highly robust and suitable choice for selective recoupling experiments, in some sense complementary to the original $\mathrm{C}_{2}^{1}$ version.

So far only the pulse durations $\tau_{1}$ and $\tau_{2}$ have been considered in the discussion due to their high impact on the experimental performance. Optimising all parameters $\left(\kappa_{1}, \kappa_{2}, \tau_{1}, \tau_{2}, \phi_{1}, \phi_{2}\right.$, $n_{\mathrm{C}}$ ) results in the overall best DQF efficiency (65.4 percent) of the GA optimisation (Table 11) and the corresponding buildup curves are shown in Figure 12. Comparison of the buildup curve for this best solution and the ideal $\mathrm{C}_{2}^{1}$ buildup (assuming absence of CSA, Figure $12 \mathrm{a}$ ) shows very good agreement between the two. However, a slightly lower buildup-rate for the optimised sequence is observed. This is mostly due to the nature of the fitness function (Equation 7) chosen such that the DQF efficiency is optimised. Improved results might be achieved by using a multi-objective fitness function, by parametrising DQF efficiency and buildup rate together. The fully optimised solution shows the very high robustness of the fully optimised solution to different magnitudes of CSA interactions. However, it also is found that the fully optimised solution has a even more narrowbanded profile of its recoupling performance as compared to the optimisation of $\tau_{1}$ alone. This narrowbandedness reflects the fact that the used fitness function does not penalise a narrowbanded characteristic of the resulting pulse sequence.

\subsection{Comparison of GA with other Algorithms}

Due to their stochastic nature, GAs have many traits of sampling the search space at random points, or of complete sampling of the search space at a given grid size. The optimum parameters shown in Table 1 are each the best result of $30 \mathrm{GA}$ runs, with each run limited to 1500 fitness function evaluations. The same optimisation is now iterated for 1500 randomly sampled points in search space for 30 runs (see Table 3). Comparison of the resulting DQF efficiencies shows that for small numbers of optimisation parameters there is no difference between random sampling and GA performance, given that 1500 points probe the search space sufficiently accurately. However, with increasing numbers of parameters the probing gets coarser and the deviation between random 


\begin{tabular}{cccccccccc}
\hline run & $\tau_{1} /[\mathrm{\mu s}]$ & $\tau_{2} /[\mathrm{\mu s}]$ & $\kappa_{1} /[\mathrm{Hz}]$ & $\kappa_{2} /[\mathrm{Hz}]$ & $\phi_{1} /\left[^{\circ}\right]$ & $\phi_{2} /\left[^{\circ}\right]$ & $n_{\mathrm{CN}} /[\#]$ & $f /[0-2]$ & $\mathrm{DQF} /[\%]$ \\
\hline \hline $\mathrm{C} 7{ }_{2}^{1}$ & 14.00 & 14.00 & 71428 & 71428 & 0.00 & 0.00 & 31 & 0.91 & 8.69 \\
\hline random & 14.36 & & & & & & & 0.44 & 55.69 \\
random & 13.97 & 14.27 & & & & & & 0.42 & 58.38 \\
random & & & 44323 & 75884 & & & & 0.69 & 30.90 \\
random & & & & & -7.95 & 147.37 & & 0.87 & 13.47 \\
random & 13.88 & 14.38 & 74524 & 70358 & & & & 0.43 & 56.64 \\
random & 14.46 & 14.02 & 66234 & $=\kappa_{1}$ & & & 32 & 0.47 & 52.59 \\
random & 14.87 & 13.38 & 64452 & 72880 & 9.70 & -2.67 & & 0.43 & 56.87 \\
random & 14.62 & 14.83 & 78208 & 75878 & 1.26 & -4.99 & 38 & 0.38 & 62.29 \\
Simplex & 14.61 & 15.01 & 76504 & 76215 & -1.86 & -8.17 & 34 & 0.34 & 65.73 \\
\hline
\end{tabular}

Table 3: Optimum parameter sets from random sampling. Empty cells correspond to values of $\mathrm{C}_{2}^{1}$; Values are best of 30 repeated GA runs; each comprising 1500 fitness evaluations, spread over population sizes of 50 individuals evolved for 30 generations. Crossover probability was $p_{\mathrm{c}}=0.6$ and mutation probability $p_{\mathrm{m}}=0.01$. Random sampling utilised parameter boundaries centred at $\mathrm{C} 7{ }_{2}^{1}$ default parameters $\left(\Delta \tau_{i}= \pm 5 \mu \mathrm{s}, \Delta \kappa_{i}= \pm 7142 \mathrm{~Hz}, \Delta \phi_{i}= \pm 10^{\circ}, \Delta n_{\mathrm{C} 7}= \pm 20\right)$

sampling and GA best result increases. One might argue that the stochastic nature of the two approaches makes the best result of 30 runs possibly merely a matter of luck. A well behaved algorithm, however, should also display a good chance to reproduce a successful search at a reasonable rate. This can be assessed e.g. by considering every run finding a DQF efficiency larger than 50 percent a success. For the scenario where all parameters are optimised simultaneously the GA has a 50 percent chance of success while random sampling only has a 17 percent chance of success.

For comparison of the GA to non-heuristic search algorithms MIGRAD [29] and Simplex [57] are chosen, representing the classes of gradient and non-gradient based search algorithms. Heuristic (GA) searches gain from large numbers of fitness-function evaluations due to the concomitant increase in population and generation sizes. Non-heuristic methods are affected by a fitness-function evaluation limit to a much lesser extend. These algorithms strongly dependent on a good initial guess of starting parameter values and on the structure of the fitness-function landscape. More specifically, the Simplex algorithm is mostly affected by the existence of local maxima in the vicinity of its starting point, but is quite independent of the shape of the maximum. The MIGRAD algorithm, like most gradient based algorithms, assumes that the maximum region can be approximated by a quadratic function and hence takes advantage of the definition of the fitness function and, in turn, tends to converge faster. MIGRAD and Simplex are unconstrained and take confidence parameters for every parameter instead. These control the variation of the optimisation parameters in the initial steps of the algorithm.

The error landscapes displayed in Figure $7 \mathrm{~b}-\mathrm{d}$ and Figure 8 can be considered as typical for NMR pulse sequence optimisation, especially for those using resonance conditions to achieve re/decoupling [60. Choosing confidence parameters identical to the parameter interval limits of the constraint GA optimisation and using the original $\mathrm{C}_{2}^{1}$ parameters as initial parameters, Simplex manages to evade the local maximum within the limit of 1500 steps, while MIGRAD fails to do so. Starting with initial parameters further away from the optimum causes both algorithms to struggle even more in achieving good efficiencies. 
As a test of the quality of the optimum parameters found by the GA, these can be passed on to additional Simplex optimisation (compare bottom of Table 1 and 3). Only a very slight further improvement (0.3 percent) is found. This can be ascribed just to the now continuous parameters as opposed to the discrete values of the GA parameters resulting from the bit-depth of encoding. Combinations of genetic algorithms and classical optimisation has been successfully exploited in other areas [65, 52, 25] for the refinement of search results but this does not seem to offer great advantages when searching for improved NMR pulse sequences. In some circumstances GA optimisations may be computationally dearer than classical algorithms but the reward may very well be the ability to find solutions not accessible to other search algorithms.

Optimal control approaches have featured prominently in recent years in searches for improved NMR pulse sequences [63, 50]. Various methods of optimal control (dynamic programming, Pontryagin minimum principle [35]) have been applied successfully using both geometrical and numerical approaches. The numerical approaches (specifically the GRAPE or Krotov implementations of optimal control [34, 45) and GA algorithms have in common that both can be applied to some extent in a black-box fashion. This makes both methods attractive tools for general applications. Apart from this, GA and optimal control have to be regarded rather complementary in their respective strengths. Due to the dependence of optimal control algorithms on gradient information they are affected by the shape of search landscapes and initial parameter values in quite the same way as are classical gradient based algorithms, where the presence of local minima may pose problems. GAs are better placed to handle such circumstances successfully. If the search space is rather smooth and optimum conditions can be reached with a continuous increase of pulse sequence performance from nearly every initial condition it may well be the case that optimal control approaches converge faster than GA searches. In searches where the optimum condition is a fairly sharp, singular resonance condition (such as is common for recoupling MAS NMR experiments) GA searches may be advantageous. GAs and optimal control approaches, in principle, have in common that both may produce results that make physical insight difficult. As far as optimal control is concerned, the need to simplify and clarify optimisation results has been recently addressed by introducing smoothing constraints to successive pulses [44] and modularisation of the pulse sequence [49] in order to aide the generation of structured results that later can be rationalised by theory. Guiding GA searches by biasing the algorithms toward certain structured results is certainly also possible. In other areas of application for optimisation procedures, it has been suggested to combine GA and optimal control algorithms for situations where the structure of the search space can not be handled well by only one of the two approaches [58, 55, 68, 1, 46].

\section{Experimental}

${ }^{31} \mathrm{C}$ NMR spectra spectra were recorded using a Bruker Avance II $700\left(\omega_{0} / 2 \pi=-176.1 \mathrm{MHz}\right)$ spectrometer equipped with a $2.5 \mathrm{~mm}$ TriGamma MAS probe. MAS spinning speed was $10204 \mathrm{~Hz}$ throughout. Heteronuclear ${ }^{1} \mathrm{H}$ decoupling was suspended during $\mathrm{C} 7$ irradiation [28, 43, during acquisition $71.4 \mathrm{kHz}{ }^{1} \mathrm{H} \mathrm{cw}$ decoupling was applied. Hartmann-Hahn cross-polarisation (CP) contact time was $5 \mathrm{~ms}$. 32 scans were accumulated per spectrum with $20 \mathrm{~s}$ recycle delays. $2 \pi$-pulse r.f. amplitudes were calibrated on 1 . After $\mathrm{CP}$, the ${ }^{13} \mathrm{C}$ signal of a train of $2 \pi$-pulses is maximised (comparing 1, 7 and 15 pulses). Different phase cycling schemes did not provide improvements over a basic 32-step cycle. Active temperature control of the sample $(T=298 \mathrm{~K})$ was used throughout. $1,4-{ }^{13} \mathrm{C}_{2}$-mono-ammonium maleate was co-crystallised [16] with mono-ammonium maleate $\left({ }^{13} \mathrm{C}\right.$ nat. abund.) in ratios 1:7 and 1:15 to suppress inter-molecular dipolar couplings effects on spin-pair 
behaviour (1:7 dilution found to be sufficient). Simulations used the SIMPSON [3] package. The genetic algorithm was implemented as an extension to SIMPSON's own fit function using the GAlib 66] library. The Simplex and MIGRAD algorithms were used in their SIMPSON implementation.

\section{Conclusions and Outlook}

GAs offer powerful and flexible tools for the optimisation of NMR pulse sequences. The most important step in such procedures is to derive a suitable encoding of the pulse sequence parametrisation as a genome, operated on by the algorithm. Since GAs offer huge flexibility of exposing specific NMR characteristics to the algorithm, this step requires careful choices in order to exploit the properties of GAs to their full capacity. In our example, it would not have been a clever choice to expose all pulse sequence parameters simultaneously and to equal extent as this would have led to a very large search space and impractical computational cost. However, GAs do allow one to make task-specific choices that, for example, draw from existing theoretical knowledge about C-sequences while allowing sufficient degrees of freedom to find optimised solutions outside the boundaries of the initial theoretical design principles. Our optimised solution maintains by and large the seven-fold symmetry principle of the sequence, it only slightly breaks the synchronicity of the pulse sequence with the rotation period of the sample. Wether or not, and if so, to which extent, the cyclic nature of the pulse sequence is affected depends on the spin-system properties and will differ for cases with and without CSA. Given that our optimisations yield performance improvements for both cases, one may cautiously speculate that the cyclic nature of the sequence is less important. This finding should represent a promising starting point for further theoretical investigations into nonsynchronous recoupling MAS NMR pulse sequences. To the best of our knowledge, this has not yet been explored. We note in passing that the occurrence of small deviations from perfect symmetry having major impacts is quite common in the physical world, ranging, for example, from so-called incommensurate structures in crystallography [64] to the properties of viruses [32, or the effects of minor decoherences in quantum information [33.

Open source NMR simulation software and GA programming libraries make it technically straightforward to assemble task-specific optimisation routines, far more sophisticated than the example we have discussed here. For example, multi-objective fitness functions are promising candidates for highly specific search tasks. In addition, more advanced genomic structures and genetic operators may be exploited in general. Rather specific to GA searches in NMR are the uniquely well structured characteristics of the underlying Hamiltonians describing the spin dynamics. Such a search environment may benefit hugely from GA approaches such as Grammatical Evolution and Cartesian Genetic Programming [56, 51, 47] where the algorithm itself is well structured. This, in turn, may lead not only to improved performance of pulse sequences but also to improved insight into the optimised NMR experiments.

\section{Acknowledgements}

Support of this work by the Deutsche Forschungsgemeinschaft, the Royal Society (JP080770), EPSRC grant EP/D050618/1 (SEBASE) and EP/J017515/1 (DAASE) are gratefully acknowledged. We thank the NMR Centre at Warwick University for spectrometer access, enabling preliminary experiments. We thank TIFR, Mumbai, and P. K. Madhu for hosting us and for making available generous laboratory access. 


\section{References}

[1] Bjarne Amstrup, Gabor J. Toth, Gabor Szabo, Herschel Rabitz, and Andras Loerincz. Genetic algorithm with migration on topology conserving maps for optimal control of quantum systems. The Journal of Physical Chemistry, 99(14):5206-5213, 1995.

[2] Jörn Schmedt auf der Günne. Distance measurements in spin- $1 / 2$ systems by ${ }^{13} \mathrm{C}$ and ${ }^{31} \mathrm{P}$ solid-state NMR in dense dipolar networks. Journal of Magnetic Resonance, 165(1):18-32, 2003.

[3] Mads Bak, Jimmy T Rasmussen, and Niels Chr Nielsen. SIMPSON: A general simulation program for solid-state NMR spectroscopy. Journal of Magnetic Resonance, 147(2):296-330, 2000 .

[4] M. Baldus, D. G. Geurts, and B. H. Meier. Broadband dipolar recoupling in rotating solids: a numerical comparison of some pulse schemes. Solid State Nuclear Magnetic Resonance, 11(3-4):157-168, 1998.

[5] Morten Bjerring and Niels Chr. Nielsen. Solid-state NMR heteronuclear dipolar recoupling using off-resonance symmetry-based pulse sequences. Chemical Physics Letters, 370(3-4):496503, MAR 142003.

[6] N. Boulant, D. Le Bihan, and A. Amadon. Strongly modulating pulses for counteracting rf inhomogeneity at high fields. Magnetic Resonance in Medicine, 60(3):701-708, 2008.

[7] Andreas Brinkmann, Jörn Schmedt auf der Günne, and Malcolm H. Levitt. Homonuclear zero-quantum recoupling in fast magic-angle spinning nuclear magnetic resonance. Journal of Magnetic Resonance, 156(1):79-96, 2002.

[8] Andreas Brinkmann and Mattias Edén. Second order average hamiltonian theory of symmetrybased pulse schemes in the nuclear magnetic resonance of rotating solids: Application to triplequantum dipolar recoupling. The Journal of Chemical Physics, 120(24):11726-11745, 2004.

[9] Andreas Brinkmann, Mattias Edén, and Malcolm H. Levitt. Synchronous helical pulse sequences in magic-angle spinning nuclear magnetic resonance: Double quantum recoupling of multiple-spin systems. The Journal of Chemical Physics, 112(19):8539-8554, 2000.

[10] Andreas Brinkmann and Malcolm H. Levitt. Symmetry principles in the nuclear magnetic resonance of spinning solids: Heteronuclear recoupling by generalized Hartmann-Hahn sequences. Journal of Chemical Physics, 115(1):357-384, 2001.

[11] Darren H. Brouwer, Per Eugen Kristiansen, Colin A. Fyfe, and Malcolm H. Levitt. Symmetrybased ${ }^{29} \mathrm{Si}$ dipolar recoupling magic angle spinning NMR spectroscopy: A new method for investigating three-dimensional structures of zeolite frameworks. Journal of the American Chemical Society, 127(2):542-543, 2005. PMID: 15643876.

[12] Marina Carravetta, Mattias Edén, Ole G. Johannessen, Henrik Luthman, Peter J. E. Verdegem, Johan Lugtenburg, Angelika Sebald, and Malcolm H. Levitt. Estimation of carboncarbon bond lengths and medium-range internuclear distances by solid-state nuclear magnetic resonance. Journal of the American Chemical Society, 123(43):10628-10638, 2001. PMID: 11673994. 
[13] Marina Carravetta, Mattias Edén, Xin Zhao, Andreas Brinkmann, and Malcolm H Levitt. Symmetry principles for the design of radiofrequency pulse sequences in the nuclear magnetic resonance of rotating solids. Chemical Physics Letters, 321(3-4):205-215, 2000.

[14] Lei Chen, Qiang Wang, Bingwen Hu, Olivier Lafon, Julien Trebosc, Feng Deng, and Jean-Paul Amoureux. Measurement of hetero-nuclear distances using a symmetry-based pulse sequence in solid-state NMR. Physical Chemistry Chemical Physics, 12:9395-9405, 2010.

[15] Edwin K. P. Chong and Stanislaw H. Zak. An Introduction to Optimization (Wiley-Interscience Series in Discrete Mathematics and Optimization). Wiley-Interscience, 3 edition, February 2008.

[16] Stephan Dusold, Heidi Maisel, and Angelika Sebald. Magnitudes and orientations of interaction tensors determined from rotational resonance mas nmr lineshapes of a four-13c-spin system. Journal of Magnetic Resonance, 141(1):78-90, 1999.

[17] Evan M. Fortunato, Marco A. Pravia, Nicolas Boulant, Grum Teklemariam, Timothy F. Havel, and David G. Cory. Design of strongly modulating pulses to implement precise effective hamiltonians for quantum information processing. The Journal of Chemical Physics, 116(17):7599-7606, 2002.

[18] Ray Freeman and Xili Wu. Design of magnetic resonance experiments by genetic evolution. Journal of Magnetic Resonance (1969), 75(1):184-189, 1987.

[19] S. J. Glaser, T. Schulte-Herbrüggen, M. Sieveking, O. Schedletzky, N. C. Nielsen, O. W. Sørensen, and C. Griesinger. Unitary Control in Quantum Ensembles: Maximizing Signal Intensity in Coherent Spectroscopy. Science, 280(5362):421-424, 1998.

[20] David E. Goldberg. Genetic Algorithms in Search, Optimization and Machine Learning. Addison-Wesley Longman Publishing Co., Inc., Boston, MA, USA, 1st edition, 1989.

[21] David E. Goldberg. The Design of Innovation: Lessons from and for Competent Genetic Algorithms. Kluwer Academic Publishers, Norwell, MA, USA, 2002.

[22] Helen Frances Gray and Ross James Maxwell. Genetic programming optimisation of nuclear magnetic resonance pulse shapes. In Proceedings of the First International Symposium on Medical Data Analysis, ISMDA '00, pages 242-249, London, UK, UK, 2000. Springer-Verlag.

[23] Dennis L.A.G. Grimminck, Suresh K. Vasa, W. Leo Meerts, Arno P.M. Kentgens, and Andreas Brinkmann. EASY-GOING DUMBO on-spectrometer optimisation of phase modulated homonuclear decoupling sequences in solid-state NMR. Chemical Physics Letters, 509(4-6):186-191, 2011.

[24] Christian Herbst, Jirada Herbst, Jörg Leppert, Oliver Ohlenschläger, Matthias Görlach, and Ramadurai Ramachandran. Numerical design of $\mathrm{R} N_{n}^{\nu}$ symmetry-based rf pulse schemes for recoupling and decoupling of nuclear spin interactions at high mas frequencies. Journal of Biomolecular NMR, 44:235-244, 2009. 10.1007/s10858-009-9335-x.

[25] D.Brynn Hibbert. Genetic algorithms in chemistry. Chemometrics and Intelligent Laboratory Systems, 19(3):277-293, 1993. 
[26] M. Hohwy and N. C. Nielsen. Elimination of high order terms in multiple pulse nuclear magnetic resonance spectroscopy: Application to homonuclear decoupling in solids. The Journal of Chemical Physics, 106(18):7571-7586, 1997.

[27] J.H. Holland. Adaptation in Natural and Artificial Systems. Ann Arbor: University of Michigan Press, 1975.

[28] Colan E. Hughes, Sorin Luca, and Marc Baldus. Radio-frequency driven polarization transfer without heteronuclear decoupling in rotating solids. Chemical Physics Letters, 385(5-6):435440, 2004.

[29] F. James and M. Roos. Minuit - a system for function minimization and analysis of the parameter errors and correlations. Computer Physics Communications, 10(6):343-367, 1975.

[30] Christopher P. Jaroniec, Brett A. Tounge, Chad M. Rienstra, Judith Herzfeld, and Robert G. Griffin. Recoupling of heteronuclear dipolar interactions with rotational-echo double-resonance at high magic-angle spinning frequencies. Journal of Magnetic Resonance, 146(1):132-139, 2000 .

[31] T. Karlsson, J. M. Popham, J. R. Long, N. Oyler, and G. P. Drobny. A study of homonuclear dipolar recoupling pulse sequences in solid-state nuclear magnetic resonance. Journal of the American Chemical Society, 125(24):7394-7407, 2003.

[32] T. Keef and R. and Twarock. New insights into viral architecture via affine extended symmetry groups. Computational and Mathematical Methods in Medicine, 9(3-4):221-229, 2008.

[33] Viv Kendon and Ben Tregenna. Decoherence can be useful in quantum walks. Physical Review A, 67:042315, Apr 2003.

[34] Navin Khaneja, Timo Reiss, Cindie Kehlet, Thomas Schulte-Herbrüggen, and Steffen J. Glaser. Optimal control of coupled spin dynamics: design of nmr pulse sequences by gradient ascent algorithms. Journal of Magnetic Resonance, 172(2):296-305, 2005.

[35] Donald E. Kirk. Optimal Control Theory: An Introduction. Dover Publications, April 2004.

[36] Per Eugen Kristiansen, Marina Carravetta, Wai Cheu Lai, and Malcolm H. Levitt. A robust pulse sequence for the determination of small homonuclear dipolar couplings in magic-angle spinning nmr. Chemical Physics Letters, 390(1-3):1-7, 2004.

[37] Per Eugen Kristiansen, Marina Carravetta, Jacco D. van Beek, Wai Cheu Lai, and Malcolm H. Levitt. Theory and applications of supercycled symmetry-based recoupling sequences in solidstate nuclear magnetic resonance. The Journal of Chemical Physics, 124(23):234510, 2006.

[38] David D. Laws, Hans-Marcus L. Bitter, and Alexej Jerschow. Solid-state NMR spectroscopic methods in chemistry. Angewandte Chemie International Edition, 41(17):3096-3129, 2002.

[39] Michal Leskes, Ümit Akbey, Hartmut Oschkinat, Barth-Jan van Rossum, and Shimon Vega. Radio frequency assisted homonuclear recoupling - a floquet description of homonuclear recoupling via surrounding heteronuclei in fully protonated to fully deuterated systems. Journal of Magnetic Resonance, 209(2):207-219, 2011. 
[40] Michal Leskes, P.K. Madhu, and Shimon Vega. Floquet theory in solid-state nuclear magnetic resonance. Progress in Nuclear Magnetic Resonance Spectroscopy, 57(4):345-380, 2010.

[41] M. H. Levitt. Composite pulses. In David M. Grant and Robin K. Harris, editors, Encyclopedia of Nuclear Magnetic Resonance, volume 2, pages 1396-1441. Wiley, 1996.

[42] Malcolm H. Levitt. Symmetry-based pulse sequences in magic-angle spinning solid-state nmr. In David M. Grant and Robin K. Harris, editors, Encyclopedia of Nuclear Magnetic Resonance. Volume 9, Advances in NMR, volume 9, pages 165-196. Wiley, 2002.

[43] Ildefonso Marin-Montesinos, Darren H. Brouwer, Giancarlo Antonioli, Wai Cheu Lai, Andreas Brinkmann, and Malcolm H. Levitt. Heteronuclear decoupling interference during symmetrybased homonuclear recoupling in solid-state NMR. Journal of Magnetic Resonance, 177(2):307$317,2005$.

[44] Ivan I. Maximov, Julien Salomon, Gabriel Turinici, and Niels Chr. Nielsen. A smoothing monotonic convergent optimal control algorithm for nuclear magnetic resonance pulse sequence design. The Journal of Chemical Physics, 132(8):084107, 2010.

[45] Ivan I. Maximov, Zdenĕk Tošner, and Niels Chr. Nielsen. Optimal control design of nmr and dynamic nuclear polarization experiments using monotonically convergent algorithms. The Journal of Chemical Physics, 128(18):184505, 2008.

[46] Zbigniew Michalewicz, Cezary Z. Janikow, and Jacek B. Krawczyk. A modified genetic algorithm for optimal control problems. Computers Ëamp; Mathematics with Applications, 23(12):83-94, 1992.

[47] J. F. Miller and P. Thomson. Cartesian genetic programming. In 3rd European Conference on Genetic Programming, volume 1802 of LNCS, pages 121-132. Springer, 2000.

[48] Avik Mitra, T. S. Mahesh, and Anil Kumar. NMR implementation of adiabatic SAT algorithm using strongly modulated pulses. The Journal of Chemical Physics, 128(12):124110, 2008.

[49] Anders Bodholt Nielsen, Morten Bjerring, Jakob Toudahl Nielsen, and Niels Chr. Nielsen. Symmetry-based dipolar recoupling by optimal control: Band-selective experiments for assignment of solid-state NMR spectra of proteins. The Journal of Chemical Physics, 131(2):025101, 2009 .

[50] Niels Chr. Nielsen, Cindie Kehlet, Steffen J. Glaser, and Navin Khaneja. Optimal control methods in nmr spectroscopy. In Encyclopedia of Magnetic Resonance. John Wiley \& Sons, Ltd, 2007.

[51] Michael O'Neill and Conor Ryan. Grammatical Evolution: Evolutionary Automatic Programming in an Arbitrary Language. Kluwer Academic Publishers, Norwell, MA, USA, 2003.

[52] Yew-Soon Ong, Meng-Hiot Lim, Ning Zhu, and Kok-Wai Wong. Classification of adaptive memetic algorithms: a comparative study. Systems, Man, and Cybernetics, Part B: Cybernetics, IEEE Transactions on, 36(1):141 -152, feb. 2006.

[53] Gaël De Paëpe, Paul Hodgkinson, and Lyndon Emsley. Improved heteronuclear decoupling schemes for solid-state magic angle spinning NMR by direct spectral optimization. Chemical Physics Letters, 376(3-4):259-267, 2003. 
[54] Yong Pang and Gary X. Shen. Improving excitation and inversion accuracy by optimized rf pulse using genetic algorithm. Journal of Magnetic Resonance, 186(1):86-93, 2007.

[55] Chungsik Park and Tai-yong Lee. Optimal control by evolutionary algorithm technique combined with spline approximation method. Chemical Engineering Communications, 191(2):262277,2004 .

[56] Riccardo Poli, William B. Langdon, and Nicholas Freitag McPhee. A Field Guide to Genetic Programming. Lulu Enterprises, UK Ltd, 2008.

[57] W.H. Press, B.P. Flannery, S.A. Teukolsky, and W.T. Vetterling. Numerical recipes in C. Cambridge University Press, Cambridge, 1992.

[58] Tarapada Roy and Debabrata Chakraborty. Genetic algorithm based optimal control of smart composite shell structures under mechanical loading and thermal gradient. Smart Materials and Structures, 18(11):115006, 2009.

[59] Dimitris Sakellariou, Anne Lesage, Paul Hodgkinson, and Lyndon Emsley. Homonuclear dipolar decoupling in solid-state NMR using continuous phase modulation. Chemical Physics Letters, 319(3-4):253-260, 2000.

[60] Ingo Scholz, Jacco D. van Beek, and Matthias Ernst. Operator-based floquet theory in solidstate nmr. Solid State Nuclear Magnetic Resonance, 37(3-4):39-59, 2010.

[61] Hans-Paul Paul Schwefel. Evolution and Optimum Seeking: The Sixth Generation. John Wiley \& Sons, Inc., New York, NY, USA, 1993.

[62] Hans Wolfgang Spiess. Double-quantum nmr spectroscopy of dipolar coupled spins under fast magic angle spinning. In Encyclopedia of Magnetic Resonance. John Wiley \& Sons, Ltd, 2007.

[63] Zdenek Tosner, Thomas Vosegaard, Cindie Kehlet, Navin Khaneja, Steffen J. Glaser, and Niels Chr. Nielsen. Optimal control in nmr spectroscopy: Numerical implementation in simpson. Journal of Magnetic Resonance, 197(2):120-134, 2009.

[64] Sander Van Smallen. Incommensurate crystal structures. Crystallography Reviews, 4(2):79202, 1995.

[65] Maria Wächtler, Annika Schweitzer, Torsten Gutmann, Hergen Breitzke, and Gerd Buntkowsky. Efficient analysis of $51 \mathrm{v}$ solid-state mas nmr spectra using genetic algorithms. Solid State Nuclear Magnetic Resonance, 35(1):37 - 48, 2009.

[66] Matthew Wall. GAlib: A C ++ library of genetic algorithm components. Mechanical Engineering Department, Massachusetts Institute of Technology, 1996.

[67] John S. Waugh. Average hamiltonian theory. In Encyclopedia of Magnetic Resonance. John Wiley \& Sons, Ltd, 2007.

[68] D. Zeidler, S. Frey, K.-L. Kompa, and M. Motzkus. Evolutionary algorithms and their application to optimal control studies. Physical Review A, 64:023420, Jul 2001. 